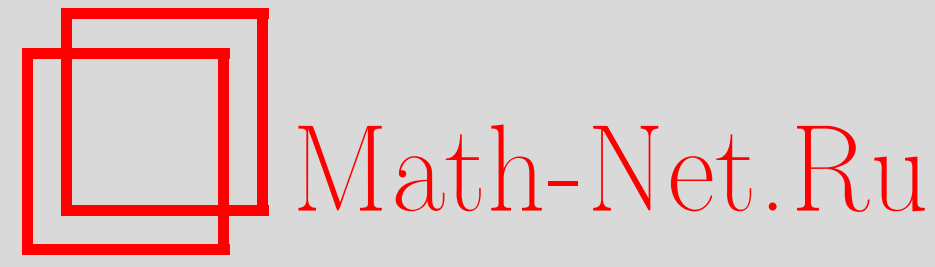

R. Kühne, L. Rüschendorf, Approximate optimal stopping of dependent sequences, Теория вероятн. и ее примен., 2003, том 48, выпуск 3, 557-575

DOI: https://doi.org/10.4213/tvp270

Использование Общероссийского математического портала MathNet.Ru подразумевает, что вы прочитали и согласны с пользовательским соглашением

http://www.mathnet.ru/rus/agreement

Параметры загрузки:

IP: 54.172 .240 .79

26 апреля 2023 г., 04:40:22

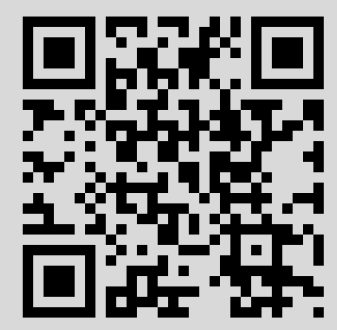


(c) 2003 г.

\author{
KÜHNE R., RÜSCHENDORF L.*
}

\title{
APPROXIMATE OPTIMAL STOPPING OF DEPENDENT SEQUENCES
}

\begin{abstract}
Рассматривается задача оптимальной остановки последовательностей случайных величин, обладающих некоторым свойством асимптотической независимости. В предположении, что вложенные точечные процессы на плоскости сходятся к процессу Пуассона, мы вводим некоторые дополнительные условия, чтобы аппроксимировать задачу оптимальной остановки последовательности с дискретным временем с помошью той же задачи для предельного процесса Пуассона. Эта предельная задача в некоторых случаях может быть решена. Мы применяем данный метод, чтобы получить аппроксимации для задач остановки последовательностей скользящего среднего, скрытых марковских цепей и последовательностей тах-авторегрессии. Кратко обсуждаются обобщения на случай, когда предельный процесс является кластерным пуассоновским.
\end{abstract}

Ключевые слова и фразы: оптимальная остановка, процессы Пуассона, асимптотическая независимость, процессы скользящего среднего, скрытые (hidden) марковские цепи.

1. Introduction. The aim of this paper is to extend the recent approach in [12] for approximatively optimal stopping of independent sequences $X_{1}, \ldots, X_{n}$ to dependent sequences. The basic assumption in this approach is convergence of the embedded planar point process

$$
N_{n}=\sum_{i=1}^{n} \varepsilon_{\left(i / n, X_{n, i}\right)} \stackrel{\mathscr{D}}{\longrightarrow} N
$$

to some Poisson point process $N$ with intensity measure $\mu$ with Lebesgue density $h$. Here $X_{n, i}=\left(X_{i}-b_{n}\right) / a_{n}$ is some normalization of $X_{i}$ induced from the central limit theorem for maxima. Then under some additional conditions it is proved that the optimal stopping problem of $X_{1}, \ldots, X_{n}$ can be approximated by the optimal stopping of the Poisson process. Finally it is shown that the optimal stopping of a Poisson process with intensity $\mu$ can

* Department of Mathematical Stochastics, University of Freiburg, Eckerstr. 1, D-79104 Freiburg, Germany; e-mail: ruschen@stochastik.uni-freiburg.de 
be reduced to solving a differential equation of the form

$$
\begin{aligned}
& v^{\prime}(t)=-\int_{v(t)}^{\infty} \int_{x}^{\infty} h(t, y) d y d x, \quad 0 \leqslant t \leqslant 1, \\
& v(1)=f(1)
\end{aligned}
$$

The solution of (1.2) is the optimal stopping curve $u$ for the Poisson process. Here $f$ is a decreasing function describing the lower boundary of the intensity and $\mu$ is assumed to have singularities only on the lower boundary; in technical terms $\mu$ is a Radon measure on $M_{f}=\{(t, y) \in[0,1] \times \overline{\mathbf{R}} ; y>f(t)\}$ supplied with the relative topology of $[0,1] \times \mathbf{R}$. Additionally to the continuity assumption (D) on $\mu$ as described above we need that $v$ satisfies the separation condition

$$
(v-f)_{/[0, t]}>c_{t}>0 \quad \text { for all } t<1 .
$$

Finally for convergence of the stopping problems we need that the positive parts $M_{n}^{+}$of the maxima $M_{n}=\max \left\{X_{1}, \ldots, X_{n}\right\}$ are uniformly integrable (condition $(\mathrm{G})$ ) and the lower curve condition (L), i.e.,

$$
\underline{\varliminf} u_{n}(1-\varepsilon)>-\infty \quad \text { for all } \varepsilon>0,
$$

where $u_{n, 1}, \ldots, u_{n, n}$ is the optimal stopping curve of $X_{n, 1}, \ldots, X_{n, n}$ and $u_{n}(s)$ $=u_{n,[n, s] \vee 1}$ is the functional form of $u_{n, i}$. Convergence of the stopping problems means convergence of the optimal stopping times, stopping time distributions, and stopping values.

To extend this program to dependent sequences we need some kind of asymptotic independence assumption (A) as well as a modification $\left(\mathrm{L}^{\prime}\right)$ of the lower curve condition.

As application we derive approximative optimal stopping results for some finite moving average processes, for hidden Markov models (chain dependent sequences), and for max-autoregressive sequences. In a subsequent section we briefly consider the case where in the limit we obtain a Poisson cluster process. This needs some alternative construction and allows to consider also some infinite moving average processes. For some details on the arguments we refer to the dissertation [11] on which this paper is based.

2. Approximation of optimal stopping. For general dependent sequences the point process approximation in (1.1) plus some conditions related to (D), (S), and (L) will not imply approximation of the stopping problems as the following simple example shows clearly.

$\mathrm{Ex} \mathrm{a} \mathrm{m} \mathrm{p} \mathrm{le} \mathrm{2.1.} \mathrm{Let}\left(Y_{i}\right)$ be i.i.d. exponentially distributed and let $T_{n}$ denote the optimal stopping time of $Y_{1}, \ldots, Y_{n}$. Let $n_{k}=10^{2^{k}}, k \geqslant 1$, and $m_{k} \in\left\{n_{k-1}+2, \ldots, n_{k}\right\}$ be such that $Y_{m_{k}}=Y_{n_{k-1}+2} \vee \cdots \vee Y_{n_{k}}$, and define

$$
X_{i}:= \begin{cases}Y_{i}, & i \neq m_{k}-1 \\ -1, & i=m_{k}-1\end{cases}
$$


Then $\left(X_{i}\right)$ exhibit the same point process behavior as $\left(Y_{i}\right)$ but have a completely different stopping behavior. Consider stopping of $\left(X_{1}, \ldots, X_{n_{k}}\right)$, then $T_{k}^{0}=\inf \left\{i \in\left\{n_{k-1}+1, \ldots, n_{k}\right\}: X_{i}=-1\right\}+1$ is a stopping time with $X_{T_{k}^{0}}=$ $X_{n_{k-1}+2} \vee \cdots \vee X_{n_{k}}$ and so $\lim _{k \rightarrow \infty}\left(\mathbf{E} X_{T_{k}^{0}}-\ln n_{k}\right)=\lim _{k \rightarrow \infty}\left(\mathbf{E} M_{n_{k}}-\ln n_{k}\right)=$ $\gamma=0.5772 \cdots>0=\lim _{k \rightarrow \infty}\left(\mathbf{E} Y_{T_{n_{k}}}-\ln n_{k}\right)$ (see [9]).

The essential local information allowing one to stop at the maximum is lost by the asymptotic approximation of the point process.

Let $\left(X_{n, i}\right)_{1 \leqslant i \leqslant n}$ be a double sequence of random variables with adapted filtration $\mathscr{F}=\left(\mathscr{F}_{n, 1}, \ldots, \mathscr{F}_{n, n}\right)$. Consider the following assumptions.

$\mathrm{C}$ ond ition (A) (asymptotic independence). Assume that for $t \in[0,1)$ there holds

$$
P^{N_{n}(\cdot \cap[t, 1] \times \mathbf{R}) \mid \mathscr{F}_{n,[n t]-1}} \stackrel{\mathbf{P}}{\longrightarrow} P^{N(\cdot \cap[t, 1] \times \mathbf{R})} .
$$

For a threshold stopping time $\tau$ with threshold $v$ define $\tau^{\geqslant t}$ as the first time over threshold $v$ after time $t$. Let $u$ denote the optimal stopping curve of the Poisson process $N$.

C o n d it i o n $\left(L^{\prime}\right)$. There exist nonincreasing constants $u_{n, i}$ such that $u_{n,[n t] \vee 1} \rightarrow u(t), t \in[0,1)$, the optimal stopping curve of the limiting Poisson process, and, for the threshold stopping time $T_{n}^{\prime}=$ $\inf \left\{i: X_{n, i} \geqslant u_{n, i}\right\}$,

$$
\lim _{t \rightarrow 1} \limsup _{n \rightarrow \infty} \mathbf{E}\left|X_{n, T_{n}^{\prime} \geqslant[n s]}\right| 1_{\left\{T_{n}^{\prime} \geqslant[n s] \geqslant[n t]\right\}}=0, \quad \text { for all } s<1 .
$$

Finally we consider the direct generalization of the lower curve condition. Define $\gamma_{n, i}=\operatorname{ess} \sup \left\{\mathbf{E}\left(X_{n, \tau} \mid \mathscr{F}_{n, i}\right) ; \tau \geqslant i\right\}$.

Condition (L).

$$
\liminf _{n \rightarrow \infty} \mathbf{E} \gamma_{n,[n t]}>-\infty, \quad \text { for all } t<1 .
$$

We now can formulate our main approximation result. Let $T$ denote the optimal stopping time of the limiting Poisson process $N=\sum \varepsilon_{\left(\tau_{k}, y_{k}\right)}$, let $K^{T}$ denote the optimal stopping index and let $T_{n}$ denote an optimal stopping time of $X_{n, i}$. (For more details on the notation see [12].)

Theorem 2.1 (approximation of stopping problems). Assume that $N_{n}=\sum_{i=1}^{n} \varepsilon_{\left(i / n, X_{n, i}\right)}$ converges on $M_{f}$ to a Poisson process $N$ with intensity $\mu$ satisfying (D). Let the optimal stopping curve $u$ be the unique solution of (1.2) and satisfy the separation condition (S). Finally assume conditions $(\mathrm{A}),(\mathrm{G})$, and $\left(\mathrm{L}^{\prime}\right)$. Then

a) $\mathbf{E}\left(\gamma_{n,[n t]} \mid \mathscr{F}_{n,[n t]-1}\right) \stackrel{\mathbf{P}}{\longrightarrow} u(t), \quad t \in[0,1)$;

b) $\left(T_{n}, X_{n, T_{n}}\right) \stackrel{\mathscr{D}}{\longrightarrow}\left(T, y_{K^{T}}\right)$ and $\mathbf{E} X_{n, T_{n}} \rightarrow \mathbf{E} y_{K^{T}}=u(0)$;

c) $\left(T_{n}^{\prime}\right)$ is an asymptotically optimal sequence of stopping times, i.e., $\mathbf{E} X_{n, T_{n}^{\prime}} \rightarrow u(0)$. 
P r o of of a). Define for $1 \leqslant \ell<m \leqslant n$

$$
M_{n, \ell, m}=X_{n, \ell} \vee \cdots \vee X_{n, m}, \quad M_{n}=M_{n, 1, n} .
$$

For $t \in[0,1)$ we define $a_{n, k}^{i}=[n(t+(1-t) i / k)], 0 \leqslant i \leqslant k, X_{n, k}^{i}=$ $M_{n, a_{n, k}^{i-1}, a_{n, k}^{i}}$ and the filtration $\mathscr{F}^{\prime}=\left(\mathscr{F}_{n, 1}^{\prime}, \ldots, \mathscr{F}_{n, k}^{\prime}\right)=\left(\mathscr{F}_{n, a_{n, k}^{1}}, \ldots, \mathscr{F}_{n, a_{n, k}^{k}}\right)$. This induces a finite stopping problem for $\bar{X}_{n, i}=X_{n, k}^{\prime j}$ if $a_{n, k}^{j-1}<i \leqslant a_{n, k}^{j}$ with corresponding filtration $\overline{\mathscr{F}}_{n, i}$ which majorizes the corresponding stopping problem of $X_{n, i}$ since $\bar{X}_{n, i} \geqslant X_{n, i}$ for all $i$. From point process convergence and the continuous mapping theorem we conclude that

$$
\left(X_{n, k}^{\prime 1}, \ldots, X_{n, k}^{\prime k}\right) \longrightarrow\left(M_{t, t+(1-t) / k}, \ldots, M_{t+(1-t)(k-1) / k, i}\right)=:\left(X_{k}^{\prime 1}, \ldots, X_{k}^{\prime k}\right)
$$

where $M_{s, t}=\sup \left\{y_{k} ; \tau_{k} \in(s, t]\right\}$ is the corresponding $\max$ in the Poisson process. Here and in the following we assume without loss of generality almost sure convergence of the points (see the corresponding argument in [12]).

We prove by backward induction from $k-1$ to 1 convergence of the optimal stopping behavior:

$$
\mathbf{E}\left(\gamma_{n, k}^{i+1} \mid \mathscr{F}_{n, i}^{\prime}\right) \stackrel{\mathbf{P}}{\longrightarrow} u_{k, i+1}^{\prime}, \quad i=0, \ldots, k-1,
$$

where $\left(u_{k, i}^{\prime}\right)$ is the optimal stopping curve of the independent sequence $\left(X_{k}^{\prime i}\right)$.

We start with the induction step $i+1 \rightarrow i$.

Consider the Bellmann equation

$$
\mathbf{E}\left(\gamma_{n, k}^{\prime i+1} \mid \mathscr{F}_{n, i}^{\prime}\right)=\mathbf{E}\left(X_{n, k}^{i+1} \vee \mathbf{E}\left(\gamma_{n, k}^{i+2} \mid \mathscr{F}_{n, i+1}^{\prime}\right) \mid \mathscr{F}_{n, i}^{\prime}\right),
$$

where by the induction assumption $\mathbf{E}\left({\gamma_{n, k}^{\prime i+2}}_{n} \mathscr{F}_{n, i+1}^{\prime}\right) \stackrel{\mathscr{D}}{\longrightarrow} u_{k, i+1}^{\prime}$. This implies conditional convergence $P^{\mathbf{E}\left(\gamma_{n, k}^{\prime i+2} \mid \mathscr{F}_{n, i+1}^{\prime}\right) \mid \mathscr{F}_{n, i}^{\prime}} \stackrel{\mathbf{P}}{\longrightarrow} \varepsilon_{u_{k, i+1}^{\prime}}$. Define the mapping $M^{0}\left(\sum \varepsilon_{\left(s_{i}, z_{i}\right)}\right)=\sup _{i} z_{i}$ and $N_{n}^{i}=\sum_{t+(1-t)(i-1) / k<j / n \leqslant t+(1-t) i / k} \varepsilon_{\left(j / n, X_{n, j}\right)}$.

Then

$$
P^{X_{n, k}^{i+1} \mid \mathscr{F}_{n, i}^{\prime}}=P^{M_{n, a_{n, k}^{i-1}+1, a_{n, k}^{i}} \mid \mathscr{F}_{n, i}^{\prime}}=P^{M^{0}\left(N_{n}^{i}\right) \mid \mathscr{F}_{n, i}^{\prime}}
$$

By the independence assumption (A),

$$
P^{N_{n}^{i} \mid \mathscr{F}_{n, i}^{\prime}} \stackrel{\mathbf{P}}{\longrightarrow} P^{N^{i}}, \quad \text { where } \quad N^{i}=\sum_{(i-1) / k<\tau_{j} \leqslant t+(1-t) i / k} \varepsilon_{\left(\tau_{j}, y_{j}\right)} .
$$

Therefore, we conclude as in $[13$, Section 4]

$$
P^{X_{n, k}^{\prime i+1} \mid \mathscr{F}_{n, i}^{\prime}} \stackrel{\mathbf{P}}{\longrightarrow} P^{M^{0} N^{i}}=P^{X_{k}^{\prime i}}
$$


For sequences of random variables $X_{n}, X$ in a separable metric space, $X_{n} \stackrel{\mathbf{P}}{\rightarrow} X$ if there exist measurable sets $m_{n}$ with $\mathbf{P}\left(m_{n}\right) \rightarrow 0$ such that pointwise

$$
X_{n} 1_{m_{n}^{c}}+X 1_{m_{n}} \rightarrow X
$$

Therefore, we obtain measurable sets $m_{n}, \mathbf{P}\left(m_{n}\right) \rightarrow 0$, such that pointwise for all $\omega$

$$
P^{N_{n}^{\prime} \mid \mathscr{F}_{n, i}^{\prime}} 1_{m_{n}^{c}}+P^{N^{i}} 1_{m_{n}} \stackrel{\mathscr{D}}{\longrightarrow} P^{N^{i}} .
$$

This implies by the continuous mapping theorem

$$
P^{M^{0} N_{n}^{\prime} \mid \mathscr{F}_{n, i}^{\prime}} 1_{m_{n}^{c}}+P^{M^{0} N^{i}} 1_{m_{n}} \stackrel{\mathscr{D}}{\longrightarrow} P^{M^{0} N^{i}}, \quad \text { i.e., } \quad P^{X_{n, k}^{\prime i+1} \mid \mathscr{F}_{n, i}^{\prime}} \stackrel{\mathbf{P}}{\longrightarrow} P^{X_{k}^{i+1}}
$$

Together, we obtain from (2.9), (2.7)

$$
P^{X_{n, k}^{\prime i+1} \vee \mathbf{E}\left(\gamma_{n, k}^{\prime i+2} \mid \mathscr{F}_{n, i+1}^{\prime}\right) \mid \mathscr{F}_{n, i}^{\prime}} \stackrel{\mathbf{P}}{\longrightarrow} P^{X_{k}^{\prime i+1} \vee u_{k, i+1}^{\prime}}
$$

To conclude from (2.10) convergence of conditional expectations as in (2.4) we next establish uniform integrability. By assumption $(G)\left(M_{n}^{+}\right)$is uniformly integrable. Therefore, also $\left(\mathbf{E}\left(M_{n}^{+} \mid \mathscr{F}_{n, i+1}^{\prime}\right)\right)$ is uniformly integrable and so with $X_{n}=\mathbf{E}\left(M_{n}^{+} \mid \mathscr{F}_{n, i+1}^{\prime}\right) 1_{\left\{\mathbf{E}\left(M_{n}^{+} \mid \mathscr{F}_{n, i+1}^{\prime}\right)>L\right\}}$ we have

$$
\lim _{L \rightarrow \infty} \limsup _{n \rightarrow \infty} \mathbf{E} X_{n}=0 \text {. }
$$

From the integrability condition $\left(\mathrm{L}^{\prime}\right)$ we conclude as in the proof of Theorem 3.2 in [12]:

$$
\lim _{n \rightarrow \infty} \mathbf{E} X_{n, T_{n}^{\prime} \geqslant[n t]}=u(t)=\mathbf{E} y_{K^{T} \geqslant t}, \quad t \in[0,1) .
$$

The positive parts of these random variables are by (2.11) uniformly integrable. Therefore, with $t=(k-1) / k$ we obtain $\left\{X_{n, T_{n}^{\prime} \geqslant[n(k-1) / k]}\right\}_{n \in \mathbf{N}}$ is uniformly integrable. This implies that $\left\{X_{n, k}^{\prime k}\right\}_{n \in \mathbf{N}}$ is uniformly integrable, again using (2.11), and, therefore,

$$
\lim _{L \rightarrow-\infty} \limsup _{n \rightarrow \infty} \mathbf{E} X_{n, k}^{\prime k} 1_{\left\{X_{n, k}^{\prime k}<L\right\}}=0, \quad \text { for any } k .
$$

Denote

$$
P_{n, \omega}=P^{X_{n, k}^{\prime i+1} \vee \mathbb{E}\left(\gamma_{n, k}^{\prime i+2} \mid \mathscr{F}_{n, i+1}^{\prime}\right)} \mid \mathscr{F}_{n, i}^{\prime}(\omega) 1_{m_{n}^{c}}(\omega)+P^{X_{k}^{\prime i+1} \vee u_{k, i+1}^{\prime}} 1_{m_{n}}(\omega)
$$

and $P_{0, \omega}=P^{X_{k}^{i+1} \vee u_{k, i+1}^{\prime}}$ and let $X_{n, i}^{\omega}, M_{n}^{\omega}$ be random variables on a probability space $\left(\Omega^{\prime}, \mathscr{A}^{\prime}, \mathbf{Q}\right)$ with $X_{n i}^{\omega} \leqslant M_{n}^{\omega}$ and

$$
\mathbf{Q}^{M_{n}^{\omega}}=P^{\mathbf{E}\left(M_{n}^{+} \mid \mathscr{F}_{n, i+1}^{\prime}\right) \mid \mathscr{F}_{n, i}^{\prime}}(\omega), \quad \mathbf{Q}^{X_{n, i}^{\omega}}=P^{\mathbb{E}\left(X_{n, k}^{\prime k} \mid \mathscr{F}_{n, i+1}^{\prime}\right) \mid \mathscr{F}_{n, i}^{\prime}}(\omega) .
$$


Then for $\omega \in m_{n}^{c}$

$\int_{L}^{\infty} x d P_{n, \omega}=\mathbf{E}\left(X_{n, k}^{i+1} \vee \mathbf{E}\left(\gamma_{n, k}^{\prime i+2} \mid \mathscr{F}_{n, i+1}^{\prime}\right) 1_{\left\{X_{n, k}^{\prime i+1} \vee \mathbf{E}\left(\gamma_{n, k}^{\prime i+2} \mid \mathscr{F}_{n, i+1}^{\prime}\right) \geqslant L\right\}} \mid \mathscr{F}_{n, i}^{\prime}\right)(\omega)$

and we obtain

$$
\begin{aligned}
\lim _{L \rightarrow \infty} \limsup _{n \rightarrow \infty} \int_{L}^{\infty} x d P_{n, \omega} \leqslant & \lim _{L \rightarrow \infty} \limsup _{n \rightarrow \infty} \mathbf{E}_{\mathbf{Q}} X_{n, i}^{\omega} 1_{\left\{X_{n, i}^{\omega} \geqslant L\right\}} \\
& +\lim _{L \rightarrow \infty} \limsup _{n \rightarrow \infty} \mathbf{E}_{\mathbf{Q}} M_{n}^{\omega} 1_{\left\{M_{n}^{\omega} \geqslant L\right\}}=0 .
\end{aligned}
$$

A similar estimate holds for the lower tail. From the uniform integrability of $P_{n, \omega}$ we conclude $\int x d P_{n, \omega} \rightarrow \int x d P_{0, \omega}$, i.e.,

$$
\mathbf{E}\left(X_{n, k}^{\prime i+1} \vee \mathbf{E}\left(\gamma_{n, k}^{\prime i+2} \mid \mathscr{F}_{n, i+1}^{\prime}\right) \mid \mathscr{F}_{n, i}^{\prime}\right) \stackrel{\mathbf{P}}{\longrightarrow} \mathbf{E}\left(X_{k}^{i+1} \vee u_{k, i+2}^{\prime}\right)
$$

This implies

$$
\mathbf{E}\left(\gamma_{n, k}^{\prime i+1} \mid \mathscr{F}_{n, i}^{\prime}\right) \stackrel{\mathscr{D}}{\longrightarrow} u_{k, i+1}^{\prime} .
$$

This proves the induction step. The beginning of the induction with $i=k-1$ is similar but simpler since the second conditional term is not present in this case.

In particular (2.4) implies $\mathbf{E}\left(\gamma_{n, k}^{\prime 1} \mid \mathscr{F}_{n, 0}^{\prime}\right) \stackrel{\mathbf{P}}{\longrightarrow} u_{k, 1}^{\prime}$. For the limit as $k \rightarrow \infty$ one obtains as in the proof of Theorem 2.5 in [12] convergence of the point process of maxima to $N$ :

$$
N^{k}=\sum_{i=1}^{k} \varepsilon_{\left(t+(1-t) i / k, X_{k}^{\prime i}\right)} \stackrel{\mathscr{D}}{\longrightarrow} N(\cdot \cap[t, 1] \times \mathbf{R}) .
$$

Define $u^{k}(t)=u_{k,[k t] \vee 1}^{\prime}, t<1 . N^{k}$ fulfills the assumptions of the approximation theorem [12, Theorem 3.2]. Therefore, we obtain $u^{k} \rightarrow u$. Since $u^{k} \geqslant u$, $\forall k$, this implies that

$$
\lim _{n \rightarrow \infty} \mathbf{P}\left\{\mathbf{E}\left(\gamma_{n,[n t]} \mid \mathscr{F}_{[n t]-1}\right) \geqslant u(t)+\varepsilon\right\}=0, \quad \text { for all } \quad \varepsilon>0
$$

Formula (2.12) and condition (G) imply $\mathbf{E}\left(\gamma_{n,[n t]} \mid \mathscr{F}_{[n t]-1}\right) \stackrel{\mathbf{P}}{\longrightarrow} u(t)$ for $t \in[0,1)$ and so a) holds.

$\mathrm{P}$ r o of of b), c). In the next step of the proof we establish convergence as in (2.4) also for the random time point $T$. Let $\left(\widetilde{T}_{n}\right)$ be a sequence of stopping time such that $\left(\widetilde{T}_{n} / n, X_{n, \widetilde{T}_{n}}\right) \stackrel{\mathscr{D}}{\longrightarrow}(T, Y)$. By a Skorokhod type argument as in [12] we assume that convergence is a.s. Our aim is to prove

$$
\mathbf{E}\left(\gamma_{n, \widetilde{T}_{n}+1} \mid \mathscr{F}_{\widetilde{T}_{n}}\right) \stackrel{\mathbf{P}}{\longrightarrow} u(T) \text { on }\{T<1\} .
$$


For the proof we use a discretization argument and define for $k \in \mathbf{N}, k \leqslant n$, $x \in[0,1)$

$$
\begin{aligned}
& g_{k}(x)=k^{-1} \inf \{i \in \mathbf{N} ; i \geqslant k x\}=[k x] k^{-1} \text { and } \\
& g_{k}^{n}(x)=\left\lceil n g_{k}\left(x n^{-1}\right)+1\right\rceil \wedge n, \quad \text { for } \quad 1 \leqslant x \leqslant n-1 .
\end{aligned}
$$

A number in $\{1, \ldots, n-1\}$ is mapped by $g_{k}^{n}$ to the nearest number of the form $\lceil n i / k+1\rceil, i=1, \ldots, k-1, n$. With $e_{k}^{n}=\left(\max _{\widetilde{T}_{n}<i<g_{k}^{n}\left(\widetilde{T}_{n}\right)} X_{n, i}-\gamma_{n, g_{k}^{n}\left(\widetilde{T}_{n}\right)}\right)_{+}$ we obtain

$$
\begin{aligned}
\mathbf{E}\left(\gamma_{n, g_{k}^{n}\left(\widetilde{T}_{n}\right)} \mid \mathscr{F}_{n, \widetilde{T}_{n}}\right) & \leqslant \mathbf{E}\left(\gamma_{n, \widetilde{T}_{n+1}} \mid \mathscr{F}_{n, \widetilde{T}_{n}}\right) \\
& \leqslant \mathbf{E}\left(\gamma_{n, g_{k}^{n}\left(\widetilde{T}_{n}\right)} \bigvee_{T_{n}<i<g_{k}^{n}\left(\widetilde{T}_{n}\right)} X_{n, i} \mid \mathscr{F}_{n, \widetilde{T}_{n}}\right) \\
& =\mathbf{E}\left(\gamma_{n, g_{k}^{n}\left(\widetilde{T}_{n}\right)} \mid \mathscr{F}_{n, \widetilde{T}_{n}}\right)+\mathbf{E}\left(e_{k}^{n} \mid \mathscr{F}_{n, \widetilde{T}_{n}}\right) .
\end{aligned}
$$

Since $\lim _{k \rightarrow \infty} g_{k}(x)=x, \forall x \in[0,1)$, and $g_{k}$ attains only $k$ values we obtain from (2.4)

$$
\mathbf{E}\left(\gamma_{n, g_{k}^{n}\left(\widetilde{T}_{n}\right)} \mid \mathscr{F}_{n, \widetilde{T}_{n}}\right) \longrightarrow u\left(g_{k}(T)\right) \quad \text { on } \quad\left\{T \leqslant \frac{k-1}{k}\right\}
$$

and, therefore,

$$
\lim _{k \rightarrow \infty} \lim _{n \rightarrow \infty} \mathbf{E}\left(\gamma_{n, g_{k}^{n}\left(\widetilde{T}_{n}\right)} \mid \mathscr{F}_{n, \widetilde{T}_{n}}\right)=u(T) \quad \text { on } \quad\{T<1\} .
$$

Consider next the behavior of $e_{k}^{n}$. We state

$$
\lim _{k \rightarrow \infty} \limsup _{n \rightarrow \infty} \mathbf{P}\left\{\mathbf{E}\left(e_{k}^{n} \mid \mathscr{F}_{n, \widetilde{T}_{n}}\right) 1_{\left\{\widetilde{T}_{n}<n(k-1) / k\right\}} \geqslant \varepsilon\right\}=0 \quad \text { for all } \varepsilon>0 .
$$

Note that $e_{k}^{n} \underset{n \rightarrow \infty}{\longrightarrow} e_{k}=\left(\max _{T<\tau_{i}<g_{k}(T)} y_{i}-u\left(g_{k}(T)\right)\right)_{+}$. For almost all $\omega \in \Omega$ there exists $k_{\omega} \in \mathbf{N}$ such that

$$
\#\left\{\left(\tau_{i}(\omega), y_{i}(\omega)\right) ; T(\omega)<\tau_{i}(\omega) \leqslant g_{k}(T(\omega)), y_{i}(\omega)>y_{K^{T(\omega)}}\right\}=0
$$

for $k \geqslant k_{\omega}$. Otherwise there would exist infinitely many points $\tau_{i}(\omega)$ close to $T(\omega)$ such that $y_{i}^{\omega}>u\left(\tau_{i}(\omega)\right)$ in contradiction to the separation assumption on the Poisson process $N$. This implies $e_{k} \rightarrow 0$ a.s., as $k \rightarrow \infty$.

By the first part of this proof $\left(\gamma_{n,[n t]}\right)_{n \in \mathbf{N}}$ is uniformly integrable. Since $\left(M_{n}^{+}\right)_{n \in \mathbf{N}}$ is uniformly integrable $\left(\mathbf{E}\left(e_{k}^{n} \mid \mathscr{F}_{n, \widetilde{T}_{n}}\right) 1_{\left\{\widetilde{T}_{n} \leqslant n(k-1) / k\right\}}\right)_{n \in \mathbf{N}}$ is also uniformly integrable and (2.18) follows as in the first part of proof. From (2.16) we obtain

$$
0 \leqslant \mathbf{E}\left(\gamma_{n, \widetilde{T}_{n+1}} \mid \mathscr{F}_{n, \widetilde{T}_{n}}\right)-\mathbf{E}\left(\gamma_{n, g_{k}^{n}\left(\widetilde{T}_{n}\right)} \mid \mathscr{F}_{n, \widetilde{T}_{n}}\right) \leqslant \mathbf{E}\left(e_{k}^{n} \mid \mathscr{F}_{n, \widetilde{T}_{n}}\right)
$$


Thus

$$
\begin{aligned}
\lim _{k \rightarrow \infty} \limsup _{n \rightarrow \infty} \mathbf{P}\{ & \left(\mathbf{E}\left(\gamma_{n, \widetilde{T}_{n+1}} \mid \mathscr{F}_{n, \widetilde{T}_{n}}\right)-\mathbf{E}\left(\gamma_{n, g_{k}^{n}\left(\widetilde{T}_{n}\right)} \mid \mathscr{F}_{n, \widetilde{T}_{n}}\right)\right) \\
& \left.\times 1_{\left\{\widetilde{T}_{n} \leqslant n(k-1) / k\right\}} \geqslant \varepsilon\right\}=0,
\end{aligned}
$$

for all $\varepsilon>0$. So with (2.17) this implies (2.14).

In the final step we prove convergence of the optimal stopping time $T_{n}$. Define $\widehat{T}_{n}=\inf \left\{i: X_{n, i} \geqslant u(i / n)\right\}$. We state that

$$
\mathbf{P}\left\{\widehat{T}_{n}=T_{n}\right\} \rightarrow 1 \text {. }
$$

By the approximation result for threshold stopping times [12, Proposition 2.4],

$$
\left(n^{-1} \widehat{T}_{n}, X_{n, \widehat{T}_{n}}\right) \stackrel{\mathscr{D}}{\longrightarrow}\left(T, y_{K^{T}}\right) .
$$

Assuming a.s. convergence and $T<1$, we obtain by (2.14)

$$
\mathbf{E}\left(\gamma_{n, \widehat{T}_{n}+1} \mid \mathscr{F}_{\widehat{T}_{n}}\right) \stackrel{\mathbf{P}}{\longrightarrow} u(T) .
$$

By the continuity assumption (D) $y_{K^{T}}>u(T)$. Then for $\omega \in \Omega$ and $n \geqslant n_{\omega}$, $X_{n, \widehat{T}_{n}}(\omega) \geqslant\left(2\left(y_{K^{T}}-u(T)\right) / 3+u(T)\right)(\omega)$. From (2.21) for $n \geqslant n_{\omega}^{\prime}$

$$
\mathbf{E}\left(\gamma_{n, \widehat{T}_{n}+1} \mid \mathscr{F}_{\widehat{T}_{n}}\right)(\omega) \leqslant\left(\frac{y_{K^{T}}-u(T)}{3}+u(T)\right)(\omega) .
$$

Together this implies for $n \geqslant n_{\omega} \vee n_{\omega}^{\prime}: X_{n, \widehat{T}_{n}}(\omega)>\mathbf{E}\left(\gamma_{n, \widehat{T}_{n}+1} \mid \mathscr{F}_{\widehat{T}_{n}}\right)(\omega)$, i.e.,

$$
\mathbf{P}\left\{\widehat{T}_{n} \geqslant T_{n}\right\} \rightarrow 1 \text {. }
$$

Conversely, suppose for some subsequence $\left(n^{\prime \prime}\right)$

$$
\mathbf{P}\left\{\widehat{T}_{n^{\prime \prime}}>T_{n^{\prime \prime}}\right\} \rightarrow d>0 .
$$

Then with some subsequence $\left(n^{\prime}\right) \subset\left(n^{\prime \prime}\right)$ such that $\left(T_{n^{\prime}} / n^{\prime}, X_{n^{\prime}, T_{n^{\prime}}}\right)$ converges and $T_{n^{\prime}} / n^{\prime} \rightarrow T^{\prime}$ we obtain as in the proof of $(2.22), \mathbf{P}\left\{T_{n^{\prime}} \geqslant \widehat{T}_{n^{\prime}}\right\} \rightarrow 1$ in contradiction to (2.23). This implies (2.19).

Asymptotic optimality of $T_{n}^{\prime}$ follows similarly to the proof of the approximation theorem in [12, Theorem 4.5]. Thus the proof of a), b), c) is complete.

A useful result to establish the point process convergence in Theorem 2.1 is the following theorem. A simple proof of this result was given in [11] based on the conditioning argument as in [7], [3]. 
Theorem 2.2 (point process convergence; see [6, Theorem 3.1]). Let $\left(X_{n, i}\right)$ be a sequence of random variables with filtration $\left(\mathscr{F}_{n, i}\right), \mu$ a measure on $[0,1] \times(a, \infty)$, and for all $x>a$ with $\mu([0,1] \times\{x\})=0$ holds:

$$
\begin{aligned}
& \sum_{i=1}^{[n t]} \mathbf{P}\left\{X_{n, i}>x \mid \mathscr{F}_{n, i-1}\right\} \stackrel{\mathbf{P}}{\longrightarrow} \mu([0, t] \times[x, \infty)), \\
& \sup _{1 \leqslant i \leqslant n} \mathbf{P}\left\{X_{n, i}>x \mid \mathscr{F}_{n, i-1}\right\} \stackrel{\mathbf{P}}{\longrightarrow} 0 .
\end{aligned}
$$

Then $N_{n}=\sum \varepsilon_{\left(i / n, X_{n, i}\right)} \stackrel{\mathscr{D}}{\longrightarrow} N$ a Poisson process on $[0,1] \times(a, \infty)$ with intensity $\mu_{/[0,1] \times(a, \infty)}$.

3. Optimal stopping of finite moving average processes. Let

$$
X_{i}=\sum_{j=1}^{k} c_{j} Y_{i-j}, \quad i \in \mathbf{N}
$$

denote a finite moving average (MA) process, where $\left(Y_{i}\right)$ are i.i.d. random variables with distribution function $F$ in the domain of an extreme value distribution $\Lambda, \Psi_{\alpha}$ or $\Phi_{\alpha}$. Point process convergence of MA-processes has been intensively studied. We will investigate approximatively optimal stopping of MA-sequences in some of these cases.

3.1. $F \in D\left(\Psi_{\alpha}\right)$.

Theorem 3.1. Let $k \in \mathbf{N}$, let $F \in D\left(\Psi_{\alpha}\right), \alpha>0$, be integrable with right endpoint of the support $\omega_{F}=0$ and assume $c_{j}>0$. Then we obtain with $a_{n}=F^{-1}\left(n^{-1 / k}\right) \Gamma(\alpha k+1)^{1 /(\alpha k)} \Gamma(\alpha+1)^{-1 / \alpha} \prod_{i=1}^{k} c_{i}^{1 / k}$ convergence of the optimal stopping problem:

a) $\mathbf{E} \frac{X_{T_{n}}}{a_{n}} \longrightarrow-\left(\frac{\alpha k}{\alpha k+1}\right)^{-1 /(\alpha k)}$;

b) $\mathbf{P}\left\{\frac{X_{T_{n}}}{a_{n}} \leqslant x\right\} \rightarrow\left\{\begin{array}{l}1, \quad x \geqslant 0, \\ 1-(-x)^{\alpha k} \frac{1}{2+1 /(\alpha k)}, \\ 0>x \geqslant-\left(\frac{\alpha k}{\alpha k+1}\right)^{-(\alpha k)^{-1}}, \\ \left(\frac{\alpha k+1}{\alpha k}\right)^{(\alpha k+1) /(\alpha k)}(-x)^{-\alpha k-1}, \\ x<-\left(\frac{\alpha k}{\alpha k+1}\right)^{-(\alpha k)^{-1}} ;\end{array}\right.$

c) $T_{n}^{\prime}=\inf \left\{i \leqslant n: X_{i} \geqslant w_{n-i}\right\}$ with $w_{n}=a_{n}\left(\alpha k /(\alpha k+1)^{-1 /(\alpha k)}\right)$ defines an asymptotically optimal sequence of stopping times. 
P r o of. For the proof we apply Theorem 2.1.

1) Point process convergence. By [5]

$$
\sum_{i=1}^{n} \varepsilon_{\left(i / n, X_{i} / \tilde{a}_{n}\right)} \stackrel{\mathscr{D}}{\longrightarrow} \tilde{N}
$$

where $\tilde{a}_{n}=F^{-1}\left(n^{-1 / k}\right)$. The point process $\tilde{N}$ is a Poisson process with intensity $\lambda_{[0,1]} \otimes \nu_{k}, \nu_{k}([x, 0])=c(\alpha, k)(-x)^{\alpha k}, x \leqslant 0$, where $c(\alpha, k)=\Gamma(\alpha+$ $1)^{k} /\left(\Gamma(k \alpha+1) \prod_{i=1}^{k} c_{i}^{\alpha}\right)$. Replacing $\tilde{a}_{n}$ by $a_{n}$, the point process converges to a Poisson point process with intensity $\lambda_{[0,1]} \otimes \nu^{\prime}, \nu^{\prime}([x, 0])=(-x)^{\alpha k}, x \leqslant 0$.

2) Condition (G) is trivially satisfied, as $M_{n}^{+} / a_{n}=0$ for all $n$.

3) (D) and uniqueness of the optimal stopping curve of $N$ by the differential equation in (1.2) has been proved in [12, Theorem 4.5]. Note that $f \equiv-\infty$ in this case.

4) Asymptotic independence (A). Note that $P^{N_{n}(\cdot \cap[t, 1] \times \mathbf{R}) \mid X_{1}, \ldots, X_{[n t]-1}}=P^{\sum_{i=[n t]}^{[n t]+k} \varepsilon_{\left(i / n, X_{i} / a_{n}\right)}+\sum_{i=[n t]+k+1}^{n} \varepsilon_{\left(i / n, X_{i} / a_{n}\right) \mid X_{1}, \ldots, X_{[n t]-1}} .}$ By the normalization we have that $\sum_{i=[n t]}^{[n t]+k} \varepsilon_{\left(i / n, X_{i} / a_{n}\right)} \stackrel{\mathscr{D}}{\longrightarrow} 0$, where 0 denotes the zero-measure. Also

$$
P^{\sum_{i=[n t]+k+1}^{n} \varepsilon_{\left(i / n, X_{i} / a_{n}\right)} \mid X_{1}, \ldots, X_{[n t]-1}}=P^{\sum_{i=[n t]+k+1}^{n} \varepsilon_{\left(i / n, X_{i} / a_{n}\right)}} \stackrel{\mathscr{D}}{\longrightarrow} P^{N_{[t, 1]}},
$$

which implies $P^{N_{n \mid[t, 1]} \mid X_{1}, \ldots, X_{[n t]-1}} \stackrel{\mathbf{P}}{\longrightarrow} P^{N_{[t, 1]}}$.

5) Condition $\left(\mathrm{L}^{\prime}\right)$. For $\ell=1, \ldots, k$ define

$$
T_{n}^{\ell}=\inf \left\{i \leqslant n: i+\ell=0(\bmod k), X_{i} \geqslant w_{n-i}\right\} .
$$

Then by the independence of $X_{i}$ involved we obtain from [9]

$$
\lim _{\varepsilon \rightarrow 0} \lim _{n \rightarrow \infty} \mathbf{E} \frac{X_{T_{n}^{\ell}}}{a_{n}} 1_{\left\{T_{n}^{\ell}>n-[n \varepsilon]\right\}}=0 .
$$

This implies using $T_{n}^{\prime}=T_{n}^{1} \wedge \cdots \wedge T_{n}^{k}$ and $0 \geqslant X_{T_{n}^{\prime}} \geqslant X_{T_{n}^{1}} \wedge \cdots \wedge X_{T_{n}^{k}}$

$$
\lim _{\varepsilon \rightarrow 0} \lim _{n \rightarrow \infty} \mathbf{E} \frac{X_{T_{n}^{\prime}}}{a_{n}} 1_{\left\{T_{n}^{\prime}>n-[n \varepsilon]\right\}} \geqslant \lim _{\varepsilon \rightarrow 0} \lim _{n \rightarrow \infty} \sum_{\ell=1}^{k} \mathbf{E}\left(\frac{X_{T_{n}^{\ell}}}{a_{n}} 1_{\left\{T_{n}^{\ell}>n-[n \varepsilon]\right\}}\right)=0 .
$$

This implies $\left(\mathrm{L}^{\prime}\right)$.

From $[8$, p. 398] we use that for $F \in D(G)$

$$
n\left(1-F\left(w_{n}\right)\right) \longrightarrow x \Longleftrightarrow \frac{w_{n}-b_{n}}{a_{n}} \longrightarrow-\ln G(x) .
$$

This implies that

$$
\frac{w_{[n t]}}{a_{n}} \longrightarrow-(1-t)^{-1 / \alpha}\left(\frac{\alpha}{1+\alpha}\right)^{-1 / \alpha}=u(t),
$$

which is the optimal stopping curve in the limiting Poisson process. Therefore, $\left(T_{n}^{\prime}\right)$ is an asymptotically optimal stopping sequence and convergence in a), b) follows from Theorem 2.1. 
3.2. $F \in D(\Lambda)$. Consider $F=F_{\alpha, p} \in D(\Lambda)$, where for $p>0, \alpha \in \mathbf{R}$

$$
1-F_{\alpha, p}(x) \sim K x^{\alpha} e^{-x^{p}} \quad \text { as } \quad x \rightarrow \infty .
$$

Consider a finite MA-process $X_{i}=\sum_{j=1}^{k} c_{j} Y_{i-j}$, where $\left(Y_{i}\right)$ are i.i.d. $Y_{i} \sim F$.

Proposition 3.1 (point process convergence; [14, Theorem 6.3]). Let $F=F_{\alpha, p}$ have a density $f \in C^{1}$ with $f(x) \sim K^{\prime} x^{\alpha^{\prime}} e^{-x^{p}}, \alpha^{\prime}=\alpha+p-1$, $K^{\prime}=K p$. Assume that

$$
\limsup _{x \rightarrow \infty}\left|\frac{x D^{\prime}(x)}{D(x)}\right|<\infty
$$

where $D(x)=f(x) e^{x^{p}}$, if $x>0$, and $D(x)=f(x)$, if $x<0$, and let $a_{n}, b_{n}$ be the normalizing constants of the associated i.i.d. sequence $Z_{i}$, where $Z_{i} \stackrel{\mathrm{d}}{=} X_{i}$ (see $[14,(5.3)$ and (5.5)]). Then

$$
\sum_{i=1}^{n} \varepsilon_{\left(i / n,\left(X_{i}-b_{n}\right) / a_{n}\right)} \stackrel{\mathscr{D}}{\longrightarrow} N
$$

a Poisson process with intensity $\mu=\lambda_{[0,1]} \otimes \nu, \nu([x, \infty))=e^{-x}, x \in \mathbf{R}$, if any of the following conditions holds:

1) $c_{i}>0, \forall i$, and $e^{c x} f^{\prime}(x)$ is bounded for $x \in(-\infty, 0]$ for some $c \geqslant 0$.

2) $f(-z)$ fulfills the assumptions on $f(z)$ with some $p^{\prime}>p$ and some $\alpha^{\prime}, K^{\prime}$.

The normal distribution satisfies the assumptions above. Under additional assumptions also convergence for infinite MA-processes is proved in [14]. As consequence we obtain from Theorem 2.1.

Corollary 3.1. Suppose $F=F_{\alpha, p}$ fulfills the conditions of Proposition 3.1 , then (formally with $c=0$, see the following Remark $3.1 \mathrm{a}$ ))

1) $\quad \frac{\mathbf{E} X_{T_{n}}-b_{n}}{a_{n}} \longrightarrow-\ln (1+c)$,

2) $\mathbf{P}\left\{\frac{X_{T_{n}}-b_{n}}{a_{n}} \leqslant x\right\} \rightarrow \begin{cases}1-\frac{1}{2} \frac{e^{-x}}{1+c}, & x \geqslant-\ln (1+c), \\ \frac{1}{2} e^{x}(1+c), & x<-\ln (1+c),\end{cases}$

3) if $w_{n}$ be constants with $n\left(1-F\left(w_{n}\right)\right) \rightarrow 1$, then for any $\varepsilon \in[0,1)$

$$
\begin{aligned}
T_{n}^{\prime}=\inf \left\{i \leqslant n:\left(i \geqslant n-[n \varepsilon] \text { and } X_{i} \geqslant w_{n-i}\right)\right. \text { or } \\
\left.\quad\left(i<n-[n \varepsilon] \text { and } \frac{X_{i}-b_{n}}{a_{n}} \geqslant u^{I}\left(\frac{i}{n}\right):=\ln \frac{1-(i / n)^{1+c}}{1+c}\right)\right\}
\end{aligned}
$$

is an asymptotically optimal sequence of stopping times. 
$\mathrm{Pr}$ o of. The proof is analogous to the proof of Theorem 3.1. Condition $(\mathrm{G})$ is implied by the uniform integrability of the normalized maxima of the i.i.d. sequence and the $k$-independence of $X_{i}$.

$\mathrm{R}$ e $\mathrm{m}$ a r k 3.1. a) Note that the general formulation with $c \neq 0$ corresponds to the optimal stopping of $X_{i}+d_{i}$ with additional observation costs $d_{i}>0, d_{i} \uparrow$, where $\left(d_{n}-d_{[n t]}\right) / a_{n} \rightarrow-c \ln t$, and with normalizations $\widehat{b}_{i}=b_{i}+c_{i}$. The optimal stopping curve in the limit then is $u_{c}^{I}(t)=\ln \left(\left(1-t^{1+c}\right) /(1+c)\right)$ (see [12, Theorem 4.3]).

b) A similar result as in Corollary 3.1 (with the same stopping limits) also holds for $p=1$ if there exists exactly one index $i$ with $c_{i}=\max \left\{c_{j}\right.$, $1 \leqslant j \leqslant m\}$ and if any of the following assumptions from [14, Theorem 7.5] holds:

1) $c_{i} \geqslant 0$

2) $F(z)=O\left(e^{-|z|^{p} / \gamma}\right)$ as $z \rightarrow-\infty$ for $\gamma$ such that $\min c_{j} \gamma^{1 / p}=\max _{j} c_{j}$;

3) $F(z) \sim K|z|^{\alpha} e^{-|z|^{p} / \gamma}$ for $\gamma$ such that $\min _{j} c_{j} \gamma^{1 / p}=\max _{j} c_{j}$.

This class contains for $p=1$ the exponential distribution.

c) Related stopping results also hold true for the class $S_{r}(\gamma)$. Let $\omega_{F}=\infty$ for some distribution function $F$ on $\mathbf{R}$. Then $F \in S_{r}(\gamma)$ for some $\gamma \geqslant 0$ if $\lim _{x \rightarrow \infty}(1-F * F(x)) /(1-F(x))=d \in(0, \infty)$ and $\lim _{x \rightarrow \infty}(1-F(x-y)) /(1-F(x))=e^{\gamma y}, \forall y \in \mathbf{R}$.

$S_{r}(0)$ contains the log-normal distribution as well as the distribution function $F$ defined by $1-F(x)=e^{-x /(\ln x)^{\alpha}}, x>1, \alpha>0$.

If $F=F_{\alpha, p}, p \in(0,1)$, then $F \in S_{r}(0)$. If $F=F_{\alpha, p}, p=1, \alpha<-1$, then $F \in S_{r}(1)$ (see [4]).

Also, if $F \in D(\Lambda) \cap S_{r}(\gamma)$ is integrable $c_{j}>0$ for all $j$, then point process convergence as in (3.8) holds if $\gamma=0$ or $c_{i}=\max \left\{c_{j}\right\}$ for some unique index $i$. (In [4] in fact a convergence result for infinite MA-processes is proved.) As in Corollary 3.1 we obtain the asymptotics of the stopping problem. It seems to be difficult to establish condition $\left(L^{\prime}\right)$ for the infinite case in this example.

d) For MA-processes with polynomial tails and $F \in D\left(\Phi_{\alpha}\right)$ the limiting point processes typically are cluster Poisson processes which need a different technique and will be dealt in short in Section 6 .

4. Hidden Markov chains (chain dependent sequences). Let $\left(J_{n}\right)_{n \in \mathbf{N}}$ be an aperiodic irreducible Markov chain with $m$ states, transition matrix $\left(p_{i j}\right)_{1 \leqslant i, j \leqslant m}$ and stationary distribution $\pi_{1}, \ldots, \pi_{m}$. A stochastic process $\left(X_{i}\right)_{i \in \mathbf{N}}$ is called hidden Markov chain (or chain-dependent) if

$$
\begin{aligned}
& \mathbf{P}\left(J_{n}=j, X_{n} \leqslant x \mid J_{0}, \ldots, J_{n-1}, X_{1}, \ldots, X_{n-1}\right) \\
& \quad=\mathbf{P}\left(J_{n}=j, X_{n} \leqslant x \mid J_{n-1}\right)=p_{J_{n-1}, j} F_{J_{n-1}}(x),
\end{aligned}
$$

for some distribution functions $F_{1}, \ldots, F_{m}$. The Markov chain $J_{n}$ chooses the distribution function at the $n$th state. 
Proposition 4.1 (point process convergence; [6, Example 3.1]). Let $\left(X_{i}\right)$ be chain-dependent and assume that

$$
\lim _{n \rightarrow \infty} n \sum_{i=1}^{m} \pi_{i} \bar{F}_{i}\left(a_{n} x+b_{n}\right)=\nu(x, \infty)
$$

for some constants $a_{n}, b_{n}$ and some nondegenerate measure $\nu$ on $\mathbf{R}$. Then

$$
N_{n}=\sum_{i=1}^{n} \varepsilon_{\left(i / n,\left(X_{i}-b_{n}\right) / a_{n}\right)} \stackrel{\mathscr{D}}{\longrightarrow} N
$$

a Poisson process with intensity $\lambda_{[0,1]} \otimes \nu$, where $x \rightarrow e^{-\nu(x, \infty)}$ is a distribution function of an extreme value type.

$\mathrm{Pr}$ o of. For the proof observe that

$$
\begin{aligned}
& \sum_{i=1}^{[n t]} \mathbf{P}\left(X_{n, i}>x \mid \mathscr{F}_{n, i-1}\right)=\sum_{i=1}^{[n t]} \mathbf{P}\left(X_{i}>x a_{n}+b_{n} \mid J_{i-1}\right) \\
& \quad=\sum_{i=1}^{[n t]}\left(1-F_{J_{i-1}}\left(x a_{n}+b_{n}\right)\right)=\sum_{j=1}^{m} \sum_{i=1}^{[n t]}\left(1-F_{j}\left(x a_{n}+b_{n}\right)\right) 1_{\left\{J_{i-1}=j\right\}} \\
& \quad=\sum_{j=1}^{m} \#\left\{j: J_{i}=j, i=0, \ldots, n-1\right\}\left(1-F_{j}\left(x a_{n}+b_{n}\right)\right) \\
& \quad \sim \sum_{j=1}^{m} n t \pi_{j}\left(1-F_{j}\left(x a_{n}+b_{n}\right)\right) \sim t \nu(x, \infty) .
\end{aligned}
$$

This implies the result by applying Theorem 2.2 .

We now establish approximation of the optimal stopping problem of this class of hidden Markov chains if the limit of type $\Phi_{\alpha}$. Then the optimal limiting stopping curve is given by $u(t)=(\alpha /(\alpha-1))^{1 / \alpha}(1-t)^{1 / \alpha}$.

Theorem 4.1 (optimal stopping of hidden Markov chain). Assume condition (4.1), where $\nu([x, \infty))=e^{-x^{\alpha}}$ for some $\alpha>1$, then with $T_{n}$ the optimal stopping time of the hidden Markov chain $X_{1}, \ldots, X_{n}$ holds,

1) $\frac{\mathbf{E} X_{T_{n}}}{a_{n}} \longrightarrow\left(\frac{\alpha}{\alpha-1}\right)^{1 / \alpha}$;

2) $\mathbf{P}\left\{\frac{X_{T_{n}}}{a_{n}} \leqslant x\right\}$

$$
\longrightarrow \begin{cases}1-x^{-\alpha} \frac{1}{2-1 / \alpha}, & x \geqslant\left(\frac{\alpha}{\alpha-1}\right)^{1 / \alpha}, \\ \frac{\alpha}{2 \alpha-1}\left(\frac{\alpha-1}{\alpha}\right)^{(\alpha-1) / \alpha} x^{\alpha-1}, & 0<x<\left(\frac{\alpha}{\alpha-1}\right)^{1 / \alpha} \\ 0, & x \leqslant 0 .\end{cases}
$$


3) $T_{n}^{\prime}:=\inf \left\{1 \leqslant i \leqslant n: X_{i} \geqslant(\alpha /(\alpha-1))^{1 / \alpha}(1-i / n)^{1 / \alpha}\right\}$ defines an asymptotically optimal sequence of stopping times.

$\mathrm{P}$ r o o f. For the proof we establish the conditions of Theorem 2.1.

1) Point process convergence of $N_{n} \rightarrow N$ is stated in Proposition 4.1. Condition (D) for the intensity measure has been established in [12, Theorem 4.4, case $c=0]$ ).

2) Condition (A): $P^{N_{n \mid[t, 1]} \mid \mathscr{F}_{n}^{i}} \stackrel{\mathbf{P}}{\longrightarrow} P^{N_{\mid[t, 1]}}$, with $i=[n t-1]$. Observe that

$$
\begin{aligned}
& P^{N_{n}(\cdot \cap[0,1] \times \mathbf{R}) \mid \mathscr{F}_{n}^{i}}=P^{N_{n}(\cdot \cap[0,1] \times \mathbf{R}) \mid J_{[n t-1]}} \\
& \quad=\sum_{j=1}^{m} P^{N_{n}(\cdot \cap[0,1] \times \mathbf{R}) \mid J_{[n t-1]}=j} 1_{\left\{J_{[n t-1]}=j\right\}} \longrightarrow P^{N(\cdot \cap[0,1] \times \mathbf{R})} \quad \text { P-a.s. }
\end{aligned}
$$

For the proof we just have to repeat the arguments in the proof of Proposition 4.1 to establish point process convergence.

3) (G): $\left(M_{n}^{+} / a_{n}\right)_{n \in \mathbf{N}}$ is uniformly integrable. For the proof let for $k \in\{1, \ldots, m\},\left(Y_{i}^{k}\right)_{i \in \mathbf{N}}$ be i.i.d. with distribution function $F_{k}$ independent of $\left(Y_{i}^{j}\right)_{i \in \mathbf{N}}$ for $j \neq k$ and of $\left(J_{n}\right)_{n \in \mathbf{N}_{0}}$. Then at stage $n \quad X_{i}$ can be defined as $Y_{i}^{k}$ with probability $p_{J_{n-1}, k}$ and, therefore, $X_{i} \leqslant Y_{i}^{1} \vee \cdots \vee Y_{i}^{m}$. With $\bar{G}_{j}=1 \wedge \pi_{j}^{-1} \sum_{i=1}^{m} \pi_{i} \bar{F}_{i}$ holds $\bar{F}_{j} \leqslant \bar{G}_{j}$ and $G_{j} \in D\left(\Phi_{\alpha}\right)$. Therefore, there exist i.i.d.r.v.'s $\left(Z_{i}^{j}\right)_{j \in \mathbf{N}}, Z_{i}^{j} \sim G_{j}, Z_{i}^{j} \geqslant Y_{i}^{j}$, and $P^{Y_{i}^{j}} \in D\left(\Phi_{\alpha}\right)$. This implies uniform integrability of $\left\{a_{n}^{-1} Y_{1}^{k} \vee \cdots \vee Y_{n}^{k}\right\}, 1 \leqslant k \leqslant m$, and, therefore, of $\left(a_{n}^{-1} \bigvee_{k=1}^{m} \bigvee_{i=1}^{n} Y_{i}^{k}\right)$. Finally this implies uniform integrability of $\left(a_{n}^{-1} M_{n}^{+}\right)$.

4) Condition (L) follows from $\lim _{n \rightarrow \infty} \mathbf{E} X_{n} / a_{n}=0$, point process convergence $N_{n} \stackrel{\mathscr{D}}{\longrightarrow} N$ and [12, Theorem 4.4].

Now the statement of Theorem 4.1 follows from Theorem 2.1 and the calculations in [12, Theorem 4.4] for the limiting stopping problem.

5. $\operatorname{Max}-\mathbf{A R}(1)$ sequences. Let $\left(Y_{i}\right)$ be an i.i.d. sequence with distribution function $F \in D\left(\Psi_{\alpha}\right), \alpha>1$. Further let $\left(Z_{i}\right)$ be a sequence of independent r.v.'s, $0 \leqslant Z_{i} \leqslant 1$, independent of $\left(Y_{i}\right)$ and let $X_{0}$ be a random variable with $\mathbf{E} X_{0}^{+}<\infty$. A max-autoregressive process of order 1 $(\max -\mathrm{AR}(1))$ is defined by

$$
X_{i}=Z_{i} \max \left\{X_{i-1}, Y_{i}\right\}, \quad i \geqslant 1 .
$$

The extremes of this process were investigated in [1]. Also the clustering properties of the exceedance point process in case $F \in D\left(\Phi_{\alpha}\right)$ has been investigated in that paper (see also the references there). The cases $F \in D(\Lambda)$, $D\left(\Psi_{\alpha}\right)$ do not exhibit this clustering property and so are simpler to deal with. To derive the optimal stopping approximations we cannot apply Theorem 2.1 directly since we do not have point process convergence of the imbedded point process to a Poisson process. Instead we argue by comparison with a related point process. 
Theorem 5.1. Assume that $F \in D\left(\Phi_{\alpha}\right), \alpha>1,0 \leqslant Z_{i} \leqslant 1$, $n^{-1} \sum_{i=1}^{[n t]} \mathbf{E} Z_{i}^{\alpha} \rightarrow t d, 0 \leqslant t \leqslant 1$, and $\mathbf{E} Z_{i}^{\alpha} \leqslant \beta$ for some $\beta<1$, then with $\left(a_{n}\right)$ the max-normalization of $F$ and $\widehat{a}_{n}=a_{n} d^{\alpha}$ holds:

1) $\mathbf{E} \frac{X_{T_{n}}}{\widehat{a}_{n}} \rightarrow\left(\frac{\alpha}{\alpha-1}\right)^{1 / \alpha}$

2) $T_{n}^{\prime}:=\inf \left\{1 \leqslant i \leqslant n: X_{i} \geqslant\left(\frac{\alpha}{\alpha-1}\right)^{1 / \alpha}\left(1-\frac{i}{n}\right)^{1 / \alpha}\right\}$ defines an asymptotically optimal sequence of stopping times.

P r o of. Define $\tilde{X}_{i}:=Z_{i} Y_{i}, i \geqslant 1$, then

$$
\tilde{N}_{n}:=\sum_{i=1}^{n} \varepsilon_{\left(i / n, \widetilde{X}_{i} \hat{a}_{n}\right)} \stackrel{\mathscr{P}}{\rightarrow} N
$$

a Poisson process with intensity defined by $\mu([0, t] \times[x, \infty))=t x^{-\alpha}$.

For the proof of (5.2) we establish the condition of Theorem 2.2. Let $1-F(x)=L(x) x^{-\alpha}$ with some $L \in R V_{0}$, then

$$
\begin{aligned}
\mathbf{P}\left\{Y_{i} Z_{i}>x \widehat{a}_{n}\right\} & =\int \mathbf{P}\left\{Y_{i}>\widehat{a}_{n} \frac{x}{z_{i}}\right\} d P^{Z_{i}}\left(z_{i}\right) \\
& =\int L\left(x \frac{\widehat{a}_{n} x}{z_{i}}\right)\left(\frac{x a_{n}}{z_{i}}\right)^{-\alpha} \frac{1}{d} d P^{Z_{i}}\left(z_{i}\right) \\
& =\left(\int L\left(x \frac{\widehat{a}_{n}}{z_{i}}\right)\left(\frac{1}{z_{i}}\right)^{-\alpha} d P^{Z_{i}}\left(z_{i}\right)\right) x^{-\alpha} a_{n}^{-\alpha} \frac{1}{d} .
\end{aligned}
$$

Therefore,

$$
\begin{aligned}
\sum_{i=1}^{[n t]} \mathbf{P}\left\{Y_{i} Z_{i}>x \widehat{a}_{n}\right\} & =\sum_{i=1}^{[n t]} \int L\left(x \frac{\widehat{a}_{n}}{z_{i}}\right)\left(\frac{1}{z_{i}}\right)^{-\alpha} d P^{Z_{i}}\left(z_{i}\right) x^{-\alpha} a_{n}^{-\alpha} \frac{1}{d} \\
& \sim \frac{x^{-\alpha}}{d} \frac{1}{n} \sum_{i=1}^{[n t]} \int Z_{i}^{\alpha} d \mathbf{P} \longrightarrow t x^{-\alpha} .
\end{aligned}
$$

Finally,

$$
\sup _{1 \leqslant i \leqslant n}\left\{\mathbf{P}\left\{\tilde{X}_{i}>x \widehat{a}_{n}\right\}\right\} \leqslant \sup _{1 \leqslant i \leqslant n}\left\{\mathbf{P}\left\{Y_{i}>x \widehat{a}_{n}\right\}\right\} \longrightarrow 0 .
$$

This implies (5.2) by Theorem 2.2 on point process convergence.

The point process $N$ fulfills (D) and $\left(\widetilde{M}_{n}^{+} / a_{n}\right)_{n \in \mathbf{N}}$ is uniformly integrable. The optimal stopping curve of $N$ is $u(t)=u_{\alpha}^{\mathrm{II}}(t)=(\alpha /(\alpha-1))^{1 / \alpha}(1-$ $t)^{1 / \alpha}$ (cf. [12, Theorem 4.4]). Further, by the approximation result for optimal stopping of independent sequences, [12, Theorem 4.4],

$$
\widetilde{T}_{n}^{\prime}:=\inf \left\{i: \widetilde{X}_{i} \geqslant \widehat{a}_{n} u\left(\frac{i}{n}\right)\right\}
$$


is asymptotically optimal and

$$
\mathbf{E} \frac{X_{\widetilde{T}_{n}^{\prime}}}{\widehat{a}_{n}} \rightarrow u(0)
$$

This implies that $u(0)$ is a lower bound for the asymptotically optimal stopping value of $X_{i}$.

In the next step we prove that

$$
\limsup _{n \rightarrow \infty} \frac{1}{\widehat{a}_{n}} \mathbf{E} \gamma_{n, 1} \leqslant u(0)
$$

i.e., $u(0)$ is in fact identical with the asymptotically optimal stopping value. For the proof note that

$$
X_{[n t]} \leqslant M_{n, 0,[(n-\sqrt{n}) t]} \prod_{i=[(n-\sqrt{n}) t]}^{[n t]} Z_{i} \vee M_{n,[(n-\sqrt{n}) t]+1,[n t]}
$$

and

$$
\frac{M_{n,[(n-\sqrt{n}) t]+1,[n t]}}{\widehat{a}_{n}} \stackrel{\mathbf{P}}{\longrightarrow} 0 .
$$

From $M_{n}=X_{1} \vee Y_{2} Z_{2} \vee \cdots \vee Y_{n} Z_{n}$ and [12, Proposition 4.1] we obtain that $\left(M_{n}^{+} / a_{n}\right)_{n \in \mathbf{N}}$ is uniformly integrable. Then $\left(M_{n, 0,[(n-\sqrt{n}) t]} / a_{n}\right)$ converges in distribution and in $L^{1}$ and $\mathbf{E} \prod_{i=[(n-\sqrt{n}) t]}^{[n, t]} Z_{i} \leqslant \beta^{\sqrt{n}-1} \rightarrow 0$, which implies $\prod_{i=[(n-\sqrt{n}) t]}^{[n t]} Z_{i} \stackrel{\mathbf{P}}{\longrightarrow} 0$ since $0 \leqslant Z_{i} \leqslant 1$. Together we obtain that

$$
\frac{X_{[n t]}}{\widehat{a}_{n}} \stackrel{\mathbf{P}}{\longrightarrow} 0 .
$$

Since $M_{n,[n s],[n t]}=X_{[n s]} \vee Y_{[n s]+1} Z_{[n s]+1} \vee \cdots \vee Y_{[n t]} Z_{[n t]}$ this implies

$$
P^{M_{n,[n s],[n t]}-b_{n} \hat{a}_{n} \mid \mathscr{F}_{[n s]-1}} \stackrel{\mathscr{D}}{\longrightarrow} P^{M_{s, t}} .
$$

Convergence as in (5.7) was the basic starting step in the proof of Theorem 2.1. Following the further steps of this proof we obtain (5.5). Obviously stopping of $X_{i}$ majorizes stopping of $\tilde{X}_{i}$ and equality holds in (5.5); i.e., part 1 ) is proved.

To prove asymptotic optimality of $T_{n}^{\prime}$ we have to establish that

$$
\mathrm{E} \frac{X_{T_{n}^{\prime}}}{\widehat{a}_{n}} \rightarrow u(0)
$$

To that purpose we prove that $T_{n}^{\prime}$ only stops asymptotically at time points, where $X_{i}=\widetilde{X}_{i}$. Define $T_{n}^{\prime \prime}=\sup \left\{i \leqslant T_{n}^{\prime}: X_{i}=\widetilde{X}_{i}\right\}$. Then

$$
\mathbf{P}\left\{T_{n}^{\prime \prime}=T_{n}^{\prime}\right\} \rightarrow 1 \text {. }
$$


We prove (5.9) by contradiction. By definition $X_{T_{n}^{\prime \prime}} \geqslant X_{T_{n}^{\prime \prime}+1} \geqslant \cdots \geqslant X_{T_{n}^{\prime}}$. Without loss of generality assume that $\left(T_{n}^{\prime} / n, X_{T_{n}^{\prime}} / \widehat{a}_{n}, T_{n}^{\prime \prime} / n, X_{T_{n}^{\prime \prime}} / \widehat{a}_{n}\right) \stackrel{\mathscr{D}}{\longrightarrow}$ $\left(T^{\prime}, y^{\prime}, T^{\prime \prime}, y^{\prime \prime}\right)$, assume pointwise convergence for all $\omega \in \Omega$ and $y_{k} \neq u\left(\tau_{k}\right)$, $\forall k \in \mathbf{N}, \tau_{i} \neq \tau_{j}, i \neq j$. Let $T$ be the optimal stopping time of $N$ and let $\left(T_{n}^{\prime}(\omega)-T_{n}^{\prime \prime}(\omega)\right) / n \rightarrow 0$, then

$$
T_{n}^{\prime}(\omega)=T_{n}^{\prime \prime}(\omega) \quad \text { for all } n>n_{\omega} .
$$

For the proof note that $\widetilde{T}_{n}^{\prime} / n \rightarrow T$ and $T<1$ a.s. Since $X_{i} \geqslant \widetilde{X}_{i}$, $T_{n}^{\prime} \leqslant \tilde{T}^{\prime}$ and so $T^{\prime}<1$ a.s. This yields $T^{\prime}(\omega)=T^{\prime \prime}(\omega)$ and by continuity $y^{\prime}(\omega) \geqslant u\left(T^{\prime}(\omega)\right)$ and, therefore, $y^{\prime \prime}(\omega) \geqslant u\left(T^{\prime}(\omega)\right)$. By the first part of the proof $\left(T^{\prime \prime}(\omega), y^{\prime \prime}(\omega)\right)$ can be assumed to be a point of $N$. Therefore, we obtain from (D) that $y^{\prime \prime}(\omega)>u\left(T^{\prime \prime}(\omega)\right)$ and so $X_{T_{n}^{\prime \prime}(\omega)}^{\prime} / \widehat{a}_{n}>u\left(T_{n}^{\prime \prime}(\omega / n)\right.$ for $n>n_{\omega}$.

This implies that at time $T_{n}^{\prime \prime}(\omega)$ the condition in the definition of $T_{n}^{\prime}(\omega)$ is fulfilled, i.e., $T_{n}^{\prime}(\omega)=T_{n}^{\prime \prime}$ for $n \geqslant n_{\omega}$. Assume now that (5.8) does not hold. Then by the previous argument $\mathbf{P}\left\{T^{\prime \prime}<T^{\prime}\right\}>0$, i.e., there exists $q \in(0,1) \cap \mathbf{Q}$ with $\mathbf{P}\left\{T^{\prime \prime}<q<T^{\prime}<1\right\}>0$. Then from definition of $T_{n}^{\prime \prime}$ and the above ordering relation $\liminf _{n \rightarrow \infty} \mathbf{P}\left\{X_{[q n]} / \widehat{a}_{n}>\varepsilon\right\}>0$ for some $\varepsilon>0$ in contradiction to $(5.6)$.

Thus we obtain $T_{n}^{\prime}(\omega)=T_{n}^{\prime \prime}(\omega)$ for $n>n_{\omega}$ and, therefore, $T_{n}^{\prime}(\omega)=\widetilde{T}_{n}^{\prime}(\omega)$ for $n>n_{\omega}$ which implies asymptotic optimality of $T_{n}^{\prime}$.

6. Some extensions to Poisson cluster processes. The results in this paper can also be extended partially to the case where one has point process convergence to some Poisson cluster process, as is typical for more general MA-processes. Consider, for example, i.i.d. random variables $\left(Y_{i}\right)$ with distribution function $F \in D\left(\Phi_{\alpha}\right)$. Let $\alpha>1, c_{j} \in \mathbf{R}, j \in \mathbf{N}$, with $c_{j} \neq 0$ for at least one index $j$, say $c_{1} \neq 0$, and $\sum_{j=1}^{\infty}\left|c_{j}\right|^{\delta}<\infty$ for some $0<\delta<1$. Consider the infinite MA-process

$$
X_{i}=\sum_{j=1}^{\infty} c_{j} Y_{i+1-j}
$$

Assume any of the following conditions:

1) $c_{j} \geqslant 0$ for all $j$;

2) $c_{j} \leqslant 0$, for all $j$ and also $F(0-\cdot) \in D\left(\Phi_{\alpha}\right)$;

3) $\mathbf{P}\left\{\left|Y_{1}\right|>x\right\} \in R V_{-\alpha}$, and $\lim _{x \rightarrow \infty} \mathbf{P}\left\{Y_{1}>x\right\} / \mathbf{P}\left\{\left|Y_{1}\right|>x\right\}=p$ exists. If $p=1$ then $c_{j}>0$ for some $j$; if $p=0$ then $c_{j}<0$ for some $j$.

Under any of these conditions point process convergence holds on $[0,1] \times$ $(0, \infty)$ :

$$
N_{n}=\sum_{i=1}^{n} \varepsilon_{\left(i / n, X_{i} / \alpha_{n}\right)} \stackrel{\mathscr{D}}{\longrightarrow} N=\sum_{k=1}^{\infty} \sum_{\substack{i=1 \\ c_{i} \neq 0}}^{\infty} \varepsilon_{\left(\tau_{k}, c_{i} y_{k}\right)},
$$


where $N^{\prime}=\sum \varepsilon_{\tau_{k}, y_{k}}$ is a Poisson process with intensity $\mu=\lambda_{[0,1]} \otimes \nu$ with $\nu([x, \infty])=x^{-\alpha}($ see $[13$, Ch. 4.5$])$.

So the limiting point process $N$ is a Poisson cluster process with deterministic cluster based on the underlying Poisson process $N^{\prime}$. For the optimal stopping we will observe at stage $n$ approximatively these limiting clusters and so it is intuitively clear that essentially the underlying Poisson process determines asymptotically the optimal stopping problem. At any observed large point one should wait until the point of the cluster arrives with the biggest coefficient. This program can be made precise and the method of the proof of Theorem 2.1 can be extended to yield the following theorem which we only formulate under conditions 1), 2) (details of the argument will be given elsewhere).

Theorem 6.1. Consider the infinite moving average process in (6.1) with $F \in D\left(\Phi_{\alpha}\right), \alpha>1$ and either condition 1) or 2). Also assume without loss of generality that $\sup _{i}\left\{\left|c_{i}\right|\right\}=1$. Then we obtain

$$
\text { 1) } \begin{aligned}
& \frac{\mathbf{E} X_{T_{n}}}{a_{n}} \longrightarrow\left(\frac{\alpha}{\alpha-1}\right)^{1 / \alpha}, \\
\text { 2) } \mathbf{P}\left\{\frac{X_{T_{n}}}{a_{n}} \leqslant x\right\} & \\
\longrightarrow & \begin{cases}1-x^{-\alpha} \frac{1}{2-\frac{1}{\alpha}}, & x \geqslant\left(\frac{\alpha}{\alpha-1}\right)^{1 / \alpha}, \\
\frac{\alpha}{2 \alpha-1}\left(\frac{\alpha-1}{\alpha}\right)^{(\alpha-1) / \alpha} x^{\alpha-1}, & 0<x<\left(\frac{\alpha}{\alpha-1}\right)^{1 / \alpha}, \\
0, & x \leqslant 0 .\end{cases}
\end{aligned}
$$

Define $m=\inf \left\{i: c_{i}=\sup _{j}\left\{\left|c_{j}\right|\right\}\right\}, w=\sup \left\{i ;\left|c_{i}\right| \geqslant\left|c_{1}\right|\right\}$, then

$$
\begin{aligned}
& T_{n}^{\prime}:=\inf \left\{i \geqslant w+1: X_{i} \geqslant a_{n} \frac{c_{1}}{c_{m}} u_{\alpha}^{\mathrm{II}}\left(\frac{i}{n}\right),\right. \\
& \left.X_{i-1} \vee \cdots \vee X_{i-w}<\frac{1}{2} a_{n} \frac{c_{1}}{c_{m}} u_{\alpha}^{\mathrm{II}}\left(\frac{i}{n}\right)\right\}+m-1
\end{aligned}
$$

defines an asymptotically optimal sequence of stopping times. Here $u_{\alpha}^{\mathrm{II}}(t)=$ $(\alpha /(\alpha-1))^{1 / \alpha}(1-t)^{1 / \alpha}$.

$u^{\text {II }}$ is the optimal stopping curve of the underlying Poisson process $N^{\prime}$ and the stopping sequence $T_{n}^{\prime}$ is defined according to the idea sketched above.

The case of random clusters in the limiting process is technically more involved even if the basic idea again is simple. When reaching a large random cluster one compares the first observed point with the max expected value of the points in the cluster to come. This max however is not fixed but has to be estimated from an initial small part of the data like the first $\sqrt{n}$ of the data. 
The details are somewhat technically involved and have been done in [11] for the case of processes of the form $X_{i}=Y_{i}+Y_{i-1}$ with a random cluster of two points in the limit and $X_{i}=Y_{i}+b Y_{i-1}+Y_{i-2}$ with a three point random cluster for $F \in D(\Lambda) \cap S_{r}(1)$. The method extends to more general cases. The formulation of the asymptotically optimal stopping time is somewhat involved but the leading idea again is to reduce this problem essentially to the underlying Poisson process and modify the optimal stopping curve by consideration of the cluster structure.

\section{REFERENCES}

1. Alpuim M. T., Catkan N. A., Hüsler J. Extremes and clustering of nonstationary maxAR(1) sequences. - Stochastic Process. Appl., 1995, v. 56, № 1, p. 171-184.

2. Anderson $C$. Extreme value theory for a class of discrete distributions with applications to some stochastic processes. - J. Appl. Probab., 1970, v. 7, p. 99-113.

3. Beska M., Klopotowski A., Slominski L. Limit theorems for random sums of dependent $d$-dimensional random vectors. - Z . Wahrscheinlichkeitstheor. verw. Geb., 1982, v. 61, p. $43-57$.

4. Davis R., Resnick S.I. Extremes of moving averages of random variables from the domain of attraction of the double exponential distribution. - Stochastic Process. Appl., 1988, v. 30, № 1, p. 41-68.

5. Davis R., Resnick S.I. Extremes of moving averages of random variables with finite endpoint. - Ann. Probab., 1991, v. 19, № 1, p. 312-328.

6. Durrett R., Resnick S.I. Functional limit theorems for dependent variables. - Ann. Probab., 1978 , v. 6, № 5, p. 829-846.

7. Jakubowski A. Principle of conditioning in limit theorems for sums of random variables. - Ann. Probab., 1986, v. 14, № 3, p. 902-915.

8. Kennedy D. P., Kertz R.P. Limit theorems for threshold-stopped random variables with applications to optimal stopping. - Adv. Appl. Probab., 1990, v. 22, №2, p. 396-411.

9. Kennedy D.P., Kertz R.P. The asymptotic behavior of the reward sequence in the optimal stopping of i.i.d. random variables. - Ann. Probab., 1991, v. 19, № 1, p. 329341.

10. Kennedy D.P., Kertz R.P. Limit theorems for suprema, threshold-stopped random variables and last exits of i.i.d. random variables with costs and discounting, with applications to optimal stopping. - Adv. Appl. Probab., 1992, v. 22, № 2, p. 241266.

11. Kühne $R$. Probleme des asymptotisch optimalen Stoppens. Dissertation. Freiburg: Universität Freiburg, 1997.

12. Kühne R., Rüschendorf L. Approximation of optimal stopping problems. - Stochastic Process. Appl., 2000, v. 90, № 2, p. 301-325.

13. Resnick S.I. Extreme Values, Regular Variation, and Point Processes. New YorkBerlin: Springer-Verlag, 1987, 320 p.

14. Rootzen $H$. Extreme value theory for moving average processes. - Ann. Probab., 1986 , v. 14 , № 2 , p. 612-652.

Поступила в редакцию

15.XI.2000

Исправленный вариант

28.II. 2003 